A multi-scale fundamental investigation of moisture induced deterioration of porous asphalt concrete

Doctoral Thesis

Author(s):

Poulikakos, Lily D.

Publication date:

2011

Permanent link:

https://doi.org/10.3929/ethz-a-006381850

Rights / license:

In Copyright - Non-Commercial Use Permitted 
Diss. ETH Nr. 19500

\title{
A MULTI-SCALE FUNDAMENTAL INVESTIGTION OF MOISTURE INDUCED DETERIORATION OF POROUS ASPHALT CONCRETE
}

\author{
A Dissertation submitted to \\ ETH Zurich
}

For the degree

Doctor of Sciences

\author{
Presented by \\ LILY DARVISH POULIKAKOS \\ B.S. Arch Eng. U of Colorado USA, M.S. Civil Eng. U. of Illinois, USA \\ Date of Birth: December $3^{\text {rd }} 1958$ \\ Citizen of United States of America and Iran
}

\author{
Accepted on the recommendation of \\ Prof. Dr. Hans J. Herrmann, Examiner \\ Prof. Dr. Manfred N. Partl, Co-Examiner
}




\section{Abstract}

In this dissertation different porous asphalt (PA) materials from a number of motorways in Switzerland have been investigated at various scales.

In part I of this investigation, direct observations of porous asphalt concrete samples in their natural state using optical and electron microscopy techniques led to important information regarding the microstructure of two mixes and the relationship to their in situ performance. Well performing and sub-optimally performing pavements show evidence of suboptimal microstructure that could lead to premature failure. Microstructural changes due to different compaction methods could be observed. Laboratory and field compaction produce different samples in terms of the microstructure. Gyratory compaction had produced more microcracks in mineral aggregates as field compacted mixes. Well performing mixes used polymer modified binders, had a more homogeneous void structure with fewer elongated voids and better interlocking of the aggregates. Furthermore, well performing mixes showed better distribution of the mastic and better coverage of the aggregates with bitumen. Observations with the low vacuum environmental scanning electron microscope (ESEM) showed that SBS polymer modification in binder and in the concentration used, exists in the form of discontinuous globules and not continuous networks. A reduction in the polymer phase was observed as a result of aging and in service use.

Porous asphalt concrete due to its open structure is exposed to water and therefore susceptible to water damage. In Part II of this investigation, the effects of water, temperature and loading frequency on macro mechanical properties of porous asphalt concrete were investigated. An innovative test method namely the coaxial shear test (CAST) developed at Empa is used to mechanically test $150 \mathrm{~mm}$ diameter cylindrical cores from eight materials in dry state and while being submerged under water. Application of CAST for a twin lay porous asphalt as well as conventional porous asphalt showed a reduction in complex modulus due to fatigue loading after each thermal cycle and due to detrimental effects of water submersion. Moisture susceptibility results using CAST reflected the field inspections results and could not be obtained using conventional test methods.

In Part III of this investigation, the tensile behavior of two types of viscoelastic bituminous films confined between mineral aggregates or steel as adherends, was investigated in the brittle and ductile regimes. Uniaxial specimens were fabricated employing a prototype set up allowing construction of micro-scale thin films and visualization of failure phenomena. The effect of key parameters, namely, 
temperature $\left(23^{\circ} \mathrm{C}\right.$ and $\left.-10^{\circ} \mathrm{C}\right)$, binder type (straight run and polymer modified), adherend type (stainless steel and mineral aggregate), and water conditioning were investigated sequentially. The results show that water sensitive aggregate-binder combinations in macro $(\varnothing=150 \mathrm{~mm})$ and mega (in service) scales also displayed reduced properties in the micro scale when water conditioned. At $23^{\circ} \mathrm{C}$ ductile failure and at $-10^{\circ} \mathrm{C}$ brittle fracture was observed. At $23^{\circ} \mathrm{C}$ phenomena, such as formation of striations during tensile mechanical loading, void nucleation and growth, filamentation and large ductile flow before fracture could be witnessed. When using proper surface preparation procedures, in all types of specimen investigated at $23^{\circ} \mathrm{C}$ only cohesive failure and at $-10^{\circ} \mathrm{C}$ predominantly adhesive-cohesive failure were found.

During the micro-tensile tests, as the upper spindle slowly moves vertically, in addition to shear forces demonstrative of viscous effects, gravity and capillary forces act on the bitumen film. In order to understand the relative effect of these forces the order of magnitude of a host of relevant dimensionless groups, representing ratios of these forces are considered and discussed. The analysis of the above numbers has given a qualitative indication that the deformation in bitumen films is dominated by viscous stresses although capillary forces do play a role with negligible inertia and gravitational effects.

An important finding of this investigation was that the difference in performance of PA materials in terms of durability and failure seen in macro and mega scales, can also be observed and quantified in their micro-structure, and micro scale mechanical tests indicating that it is the micro scale where performance determining phenomena find their origin and where research focusing on performance enhancing material optimization can begin. 


\section{Zusammenfassung}

Diese Dissertation enthält eine detaillierte, wissenschaftliche Untersuchung der Struktur und des mechanischen Verhaltens von offenporigen Asphalt-Materialien (Porous Asphalt, PA) über einen breiten Bereich von unterschiedlichen Längenskalen vom Mikro- bis in den Megabereich. Die untersuchten Proben entsprechen dem in Schweizer Autobahnen verwendeten, offenporigen Asphalt.

Im ersten Teil der Dissertation ergaben direkte Beobachtungen mittels Licht- und Elektronenmikroskopie von offenporigen Asphaltbeton-Prüfkörpern im Urzustand wichtige Informationen über die Mikrostruktur von zwei Mischguten und deren Beziehung auf das in Situ-Verhalten dieser Materialien. Die Proben mit besserem in Situ-Verhalten wiesen eine gewisse „optimale“ Mikrostruktur auf, die mit verbesserten Eigenschaften verbunden war. Dagegen war eine suboptimale Mikrostruktur immer mit entsprechend suboptimalem Verhalten und Eigenschaften der gleichen Materialien verbunden. Diese suboptimalen Mikrostrukturen und Eigenschaften waren vermutlich der Grund für das frühzeitige Versagen des Materials. Es wurden Unterschiede in der Mikrostruktur festgestellt, welche durch unterschiedliche Verdichtungsmethoden entstanden waren. Die Prüfkörper, die im Labor mit dem Gyrator verdichtet worden waren, verhielten sich anders als diejenigen, welche vor Ort verdichtet worden waren. Erstere wiesen eine grössere Anzahl von Mikrorissen auf. Die Materialien mit besserem in Situ-Verhalten enthielten ein polymermodifiziertes Bindemittel und wiesen einen homogeneren Hohlraumgehalt mit einem kleinen Anteil von länglichen Hohlräumen auf, und die Zuschlagsstoffe waren besser verzahnt. Ausserdem wurde in diesen Materialien eine bessere Verteilung des Mastix und eine bessere Umhüllung der Zuschlagsstoffe festgestellt. Die Rasterelektronenmikroskopie im Tiefvakuummodus (ESEM) ergab, dass StyrolButadien -Styrol (SBS) in polymermodifizierten Bindemitteln als Globuli locker verstreut und nicht im Netzverbund vorkommen. Es wurde auch eine Reduktion der Polymerphase festgestellt, was auf die Alterung und die tägliche Belastung zurückzuführen ist.

Wegen des grossen Hohlraumgehalts ist offenporiger Asphaltbeton leichter mit Wasser in Kontakt und deshalb anfälliger für eine Schädigung durch Wasser. Im zweiten Teil dieser Dissertation wurden die Einwirkung des Wassers, der Einfluss der Umgebungstemperatur und der Belastungsfrequenz auf die makromechanischen Eigenschaften von offenporigem Asphaltbeton untersucht. Es wurde eine an der Empa entwickelte, innovative Prüfmethode, der sogennante koaxiale Schubversuch 
(CAST), verwendet, um die mechanischen Eigenschaften von ausgesuchten, zylindrischen Bohrkernen aus acht Materialien $(\varnothing=150 \mathrm{~mm})$ im trockenen und auch im wassergesättigten Zustand zu untersuchen. Im Fall des zweischichtigen sowie im Fall des konventionellen porösen Asphalts zeigte die Anwendung von CAST, dass die Reduktion des komplexen Moduls sowohl ein direktes Ergebnis der Ermüdung und Temperaturoszillationen als auch eine Folge der negativen Wirkung der Wasserlagerung sein kann. Die Ergebnisse der Wasserempfindlichkeitsuntersuchungen mittels der CAST entsprachen der in SituBeurteilung der gleichen Materialien und konnten nicht mit konventionellen methoden erreicht werden.

Der dritte Teil der Dissertation konzentriert sich auf das Zugverhalten von zwei Arten viskoelastischer Bitumenfilme, welche zwischen Zuschlagsstoffen oder Stahlteilen, die als Adhäsionsflächen wirken, eingepasst sind. Sowohl der duktile als auch der spröde Zustand wurden untersucht. Mittels einer neuartigen Methode wurden uniaxiale Prüfkörper mit dünnen (im Mikrobereich) Bitumenfilmen hergestellt und geprüft, so dass die damit verbundenen Bruchphänomene beobachtet werden konnten. Die Wirkung von Schlüsselparametern wie z.B. die Temperatur $\left(23^{\circ} \mathrm{C}\right.$ und $-10^{\circ} \mathrm{C}$ ), der Bindemitteltyp (mit und ohne Polymermodifizierung), die Adhäsionsflächen (rostfreier Stahl und Zuschlagsstoffe) und die Wasserkonditionierung wurde systematisch untersucht. Kombinationen von Zuschlagsstoffen und Bindemitteln, die im Mikrobereich eine Verminderung diverser Eigenschaften nach der Wasserkonditionierung ergeben hatten, zeigten auch ein ähnlich wasserempfindliches Verhalten im Makro- $(\varnothing=150 \mathrm{~mm}$ ) und Megabereich (in-Situ). Bei $23^{\circ} \mathrm{C}$ erfolgte ein duktiler und bei $-10^{\circ} \mathrm{C}$ ein spröder Bruch. Bei $23^{\circ} \mathrm{C}$ konnten Phänomene wie Streifenbildung während der Zugprüfung, Löcherbildung und deren Vergrösserungen, Faserbildung und grosse duktile Dehnungen vor dem Bruch beobachtet werden. Bei richtiger Oberflächenvorbereitung konnten für alle bei $23^{\circ} \mathrm{C}$ geprüften Prüfkörper nur Kohäsionsbrüche festgestellt werden, wogegen bei $-10^{\circ} \mathrm{C}$ die adhäsiv-kohäsiven Brüche überwogen.

Während der Mikrozugversuche wirkten neben den durch die vertikale mechanische Zugbeanspruchung durch viskose Effekte erzeugten Scherkräften auch die Schwerkraft und Kapillarkräfte auf den Bitumenfilm. Um den Gesamteinfluss von diesen Effekten beurteilen zu können, wurde eine Grössenordnungsanalyse der relativen Gewichtung dieser Kräfte mit Hilfe der relevanten dimensionslosen Kennzahlen durchgeführt. Die Analyse aller Testergebnisse ergab, dass die Deformation des Bitumens von viskosen Effekten dominiert ist, wobei die kapillarkräfte einen geringen und die Trägheitskräfte und die Schwerkräfte einen vernachlässigen Einfluss ausüben.

Ein wichtiges allgemeines Ergebnis dieser Untersuchung war, dass das unterschiedliche Verhalten bezüglich Dauerhaftigkeit und Versagen von PA im Makro- und Megabereich ebenfalls im Mikrobereich beobachtet und quantifiziert werden konnte. Es steht deshalb fest, dass entsprechende, konzentrierte Forschung im Mikrobereich wesentlich dazu beitragen kann, das Ziel, Strassenbeläge mit PA zu verbessern, zu realisieren. 